\title{
SOME NEW RESULTS ON THE INITIAL TRANSIENT PROBLEM
}

\author{
Peter W. Glynn \\ Department of Operations Research \\ Stanford University \\ Stanford, CA $94305-4022$, U.S.A.
}

\begin{abstract}
This paper contains two new results pertaining to the initial transient problem for steady-state simulations. Our first result rigorously establishes the asymptotic superiority of a few long replications relative to a large number of shorter replications, assuming that no initial transient deletion is attempted. Our second result concerns an initial transient detection test proposed by Schruben; we develop asymptotics that are suggestive of the types of initial transients that the test is capable of detecting. As one might expect, the ability to detect a non-stationarity in the simulation output depends both on the magnitude of the non-stationarity of the initial condition, and the degree of autocorrelation in the process.
\end{abstract}

\section{INTRODUCTION}

Let $X=(X(t): t \geq 0)$ be a real-valued stochastic process representing the output of a discrete-event simulation. We say that $X$ possesses a steady-state if there exists a (deterministic) constant $\alpha$ such that

$$
\bar{X}(t) \triangleq \frac{1}{t} \int_{0}^{t} Y(s) d s \Rightarrow \alpha
$$

as $t \rightarrow \infty$, where $\Rightarrow$ denotes convergence in distribution, and $\triangleq$ a definition. Assuming (1) holds, the parameter $\alpha$ is referred to as the steady-state mean of $X$; the steady-state simulation problem is concerned with the use of simulation to numerically compute $\alpha$.

Of course, typically the distribution under which $X$ is initiated is atypical of the steady-state of $X$, and consequently the initial portion of the simulation is not representative of the steady-state. As a result, the initial portion of the simulation contains output that is biased relative to the steady-state. The initial transient problem is concerned with the study of this bias, and the development of associated procedures that can be used to mitigate its impact.
Note that the law of large numbers (1) suggests using $\bar{X}(t)$ as an estimator for $\alpha$. To assess the rate of convergence of $\bar{X}(t)$ to $\alpha$, it is common practice to construct a confidence interval for $\alpha$ based on the estimator $\bar{Y}(t)$. In great generality, it is known that the law of large numbers (1) is typically accompanied by a central limit theorem (CLT); see, for example, Glynn and Iglehart (1990) for a discussion of this phenomenon. The CLT asserts the existence of a (deterministic) constant $\sigma$ such that

$$
t^{1 / 2}(\bar{Y}(t)-\alpha) \Rightarrow \sigma N(0,1)
$$

as $t \rightarrow \infty$, where $N(0,1)$ denotes a normal r.v. with zero mean and unit variance; the constant $\sigma^{2}$ is known as the time-average variance of $X$. In order to construct confidence intervals for $\alpha$ based on (2), it is necessary to either estimate $\sigma^{2}$ directly, or to somehow develop a limit theorem involving $\overline{\mathrm{Y}}(t)$ in which $\sigma^{2}$ is "cancelled out". Direct estimation of $\sigma^{2}$ is a special challenge in this estimation setting, since the estimator $\bar{Y}(t)$ is effectively constructed from just one replication of $X$, and consequently sophisticated methods need to be applied in order to estimate $\sigma^{2}$. Such methods include the regenerative method, spectral analysis techniques, and auto-regressive approaches; see Bratley, Fox, and Schrage (1987) for details.

To avoid this variance estimation difficulty, one can instead replicate $X \mathrm{~m}$ independent times, running each replication to time $t / m$ so that the total time horizon simulated is identical to that needed for $\bar{X}(t)$. Letting $X_{1}, X_{2}, \ldots$ be the associated replications of $X$, this leads to the multiple replications estimator

$$
\alpha_{m}(l) \triangleq \frac{1}{m} \sum_{i=1}^{m} \bar{X}_{i}(t / m)
$$

where $\bar{Y}_{i}(t) \triangleq t^{-1} \int_{0}^{t} X_{i}(s) d s$. Given that $m \geq 2$, an 
obvious estimator for the variance of $r_{m}(l)$ is then

$$
v_{m}(t) \triangleq \frac{1}{m(m-1)} \sum_{i=1}^{m}\left(\bar{X}_{i}(t / m)-\alpha_{m}(t)\right)^{2} .
$$

Of course, one difficulty with the multiple replications approach just described is that the initial transient phase is itself replicated in cach of the $m$ replications, thereby magnifying the impact of the initial transient. This suggests that a few long replications ought to be superior to many shorter replications. In section 2 , this result is rigorously established by using the criterion of mean square error (MSE) as a basis for comparison.

In section 3 , we consider an initial bias detection test proposed by Schruben (1982). We use a class of autoregressive processes to study the sensitivity of the test to the initial condition of the simulation, and autocorrelation structure of the process.

\section{THE SUPERIORITY OF A FEW LONG RUNS}

In this section, we shall analyze the asymptotic MSE of $\alpha_{m}(t)$ as $t \rightarrow \infty$. In Glynn (1987), the multiple replicates estimator $\alpha_{m}(t)$ was studied using methods of weak convergence; however, most of the theory presented there assumes that $m \rightarrow \infty$ as $t \rightarrow \infty$. In our current discussion, however, we are especially interested in the case of small to moderate values of $m$, and our tools are consequently somewhat different.

The basic assumption underlying the analysis of this section is:

A1 There exist constants $b$ and $c$, and a positive constant $\gamma$ such that

$$
E \bar{X}(t)=\alpha+\frac{b}{t}+O\left(e^{-\gamma t}\right)
$$

and

$$
\operatorname{var} \bar{X}(t)=\frac{\sigma^{2}}{t}+\frac{c}{t^{2}}+O\left(e^{-\gamma t}\right)
$$

as $t \rightarrow \infty$.

It is shown in Glynn (1984) that such asymptotic expansions hold, for example, when $X$ is a finitestate, irreducible, continuous-time Markov chain. We shall provide an alternative justification for this assumption later in this section.

With (3) and (4) at our disposal, observe that

$$
\begin{aligned}
E \alpha_{m}(t) & =E \bar{Y}(t / m) \\
& =\alpha+\frac{m b}{t}+O\left(e^{-\gamma t / m}\right)
\end{aligned}
$$

and

$$
\begin{aligned}
\operatorname{var} \alpha_{m}(t) & =\frac{1}{m} \operatorname{var} \bar{X}(t / m) \\
& =\frac{\sigma^{2}}{t}+\frac{m c}{t^{2}}+O\left(e^{-\gamma t / m}\right)
\end{aligned}
$$

as $t \rightarrow \infty$. Consequently, the MSE of $\alpha_{m}(t)$ as an estimator of $\alpha$ can be expressed as

$$
\begin{aligned}
E\left(\alpha_{m}(t)-\gamma\right)^{2} & =\operatorname{var} \alpha_{m}(t)+\left(E \alpha_{m}(t)-\alpha\right)^{2} \\
& =\frac{\sigma^{2}}{t}+\frac{m}{t^{2}}\left(m b^{2}+c\right)+O\left(e^{-\gamma t / m}\right) .
\end{aligned}
$$

We can summarize the above discussion with the following Proposition.

Proposition 1 Under A1,

$$
E\left(\alpha_{m}(t)-\alpha\right)^{2}=\frac{\sigma^{2}}{t}+\frac{m}{t^{2}}\left(m b^{2}+c\right)+O\left(e^{-\gamma t / m}\right)
$$

as $t \rightarrow \infty$.

Note that so long as $c$ is non-negative, it is clear that setting $m=1$ minimizes MSE. However, even if $c$ is negative, it is never optimal, from the standpoint of asymptotic MSE, to choose $m$ strictly greater than $\max \left(1,-c / b^{2}\right)$. Since $c$ and $b$ are typically unknown, a conservative approach to choosing $m$ sets $m=1$; the idea is that if we choose $m$ too large, the penalty for doing so can be arbitrarily bad, whereas the downside risk associated with choosing $m=1$ is an inflation in the MSE that is of asymptotic order at most $\left(4 b^{4}+\right.$ $\left.4 b^{2} c+c^{2}\right) /\left(4 b^{2} t^{2}\right)$.

We turn now to justifying A1. Many different approaches are available, but we shall focus on providing a coupling argument for A1; see Lindvall (1992) to get a sense of the scope and power of this method. In any case, we shall use coupling to prove $\mathrm{A} 1$ for a discretetime simulation output, in which $\mathrm{X}=\left(\mathrm{X}_{n}: n \geq 0\right)$ takes the form $X_{n}=f\left(Z_{n}\right)$, where $Z=\left(Z_{n}: n \geq 0\right)$ is a Markov chain taking values in some state space $S$, and $f: S \rightarrow \mathbb{R}$ is a bounded function. Let $P^{m}(x, \cdot)=P\left(Z_{m} \in \cdot \mid Z_{0}=x\right)$ be the $m$-step transition kernel of $Z$. We shall require that there exist a constant $\lambda>0, m \geq 1$, and a probability distribution $\varphi$ on $S$ such that

$$
P^{m}(x, \cdot) \geq \lambda \varphi(\cdot)
$$

for all $x \in S$; condition (5) is closely related to the concept of Doeblin recurrence. (Note that any finitestate, aperiodic, irreducible Markov chain automatically satisfies (5).) It is well known that (5) implies that $Z$ possesses a unique (steady-state) stationary distribution $\pi$; see Meyn and Tweedie (1993) for details. 
For a given function $g: S \rightarrow \mathbb{R}$, let

$$
\|g\| \triangleq \sup \{|g(x)|: x \in S\} .
$$

Also, let $Z^{*}$ be a stationary version of $Z$ (ie. a Markov chain on $S$ with the same transition law as $Z$, but initiated with the stationary distribution $\pi$ ). ('oupling plays a key role in establishing the following inequality.

Proposition 2 Suppose (5) is satisfied. Then there exists $\rho$ with $|\rho|<1$ such that

$$
\left|E f\left(Z_{i}\right) g\left(Z_{i+j}\right)-E f\left(Z_{0}^{*}\right) g\left(Z_{j}^{*}\right)\right| \leq \frac{2\|f\|\|\mid g\|}{1-\lambda} \rho^{i} .
$$

Proof: Let $Z_{0}$ and $Z_{0}^{*}$ be chosen independently according to their respective initial distributions and set $Q(x, \cdot)=\left(P^{m}(x, \cdot)-\lambda \varphi(\cdot)\right) /(1-\lambda)$. With probability $\lambda$, we can force $Z_{m}=Z_{m}^{*}$ by requiring that both chains distribute themselves according to $\varphi$; with probability $1-\lambda$, we allow $Z_{m}$ and $Z_{m}^{*}$ to distribute themselves independently according to $Q\left(Z_{0}, \cdot\right)$ and $Q\left(Z_{0}^{*}, \cdot\right)$ respectively. The intermediate values $\left(Z_{1}\right.$, $\left.\ldots, Z_{m-1}\right)$ and $\left(Z_{1}^{*}, \ldots, Z_{m-1}^{*}\right)$ are then generated independently according to their respective conditional distributions (conditional on the initial state, and the state at time $m$ ). If $Z_{m}=Z_{m}^{*}$, we then let the two chains run together henceforth, setting $Z_{n}=Z_{n}^{*}$ for $n \geq m$. Otherwise, we repeat, conditional on $\left(Z_{m}, Z_{m}^{*}\right)$, the construction above, so that with probability $\lambda, Z_{2 m}$ and $Z_{2 m}^{*}$ agree. Again, if we get agreement, we set $Z_{n}=Z_{n}^{*}$ for $n \geq 2 \mathrm{~m}$. This process gets repeated at multiples of time $m$ until we get an agreement in $Z$ and $Z^{*}$, after which we let the two chains run together. This construction establishes the existence of a coupling time $T$ such that $Z_{n}=Z_{n}^{*}$ for $n \geq T$, and $P(T>n) \leq(1-\lambda)^{\lfloor n / m\rfloor}$.

Clearly

$$
\begin{array}{rl}
\mid E & f\left(Z_{i}\right) g\left(Z_{i+j}\right)-E f\left(Z_{0}^{*}\right) g\left(Z_{j}^{*}\right) \mid \\
& =\left|E f\left(Z_{i}\right) g\left(Z_{i+j}\right)-E f\left(Z_{i}^{*}\right) g\left(Z_{i+j}^{*}\right)\right| \\
& =\left|E\left[f\left(Z_{i}\right) g\left(Z_{i+j}\right)-f\left(Z_{i}^{*}\right) g\left(Z_{i+j}^{*}\right) ; T>i\right]\right| \\
& \leq 2\|f\|\|g\| P(T>i) \\
& \leq 2\|f\|\|g\|(1-\lambda)^{\lfloor i / m\rfloor},
\end{array}
$$

proving the result.

Turning now to (3), Proposition 2 establishes that

$$
\left|E f\left(Z_{i}\right)-E f\left(Z_{0}^{*}\right)\right|=O\left(\rho^{i}\right) .
$$

Consequently,

$$
\sum_{i=0}^{\infty}\left|E f_{c}\left(Z_{i}\right)\right|<\infty
$$

where $f_{c}(x)=f(x)-E f\left(Z_{0}^{*}\right)$. Hence,

$$
\begin{aligned}
E_{n} \frac{1}{n} \sum_{j=0}^{n-1} X_{j} & =E f\left(Z_{0}^{*}\right)+\frac{1}{n} \sum_{j=0}^{n-1} E f_{c}\left(Z_{j}\right) \\
& =E f\left(Z_{0}^{*}\right)+\frac{1}{n} \sum_{j=0}^{\infty} E f_{c}\left(Z_{j}\right) \\
& -\frac{1}{n} \sum_{k=n}^{\infty} E f_{c}\left(Z_{k}\right) \\
\triangleq & E f\left(Z_{0}^{*}\right)+\frac{b}{n}+O\left(\rho^{n}\right)
\end{aligned}
$$

where $b=\sum_{j=0}^{\infty} E f_{c}\left(Z_{j}\right)$. To prove (4) requires more work. For $x \in S$, let $E_{r}(\cdot)$ denote the expectation operator conditional on $Z_{0}=x$, and set

$$
h(x)=\sum_{k=1}^{c} E_{x} f_{c}\left(Z_{k}\right) .
$$

Proposition 2 guarantees that $h$ is a bounded function (since we are assuming $f$ is). Observe that

$$
\begin{aligned}
E\left(\sum_{i=0}^{n} f_{c}\left(Z_{i}\right)\right)^{2}= & \sum_{i=0}^{n} E f_{c}^{2}\left(Z_{i}\right) \\
& +2 \sum_{i=0}^{n-1} \sum_{j=1}^{n-i} E f_{c}\left(Z_{i}\right) f_{c}\left(Z_{i+j}\right) \\
= & \sum_{i=0}^{n} E f_{c}^{2}\left(Z_{i}\right) \\
& +2 \sum_{i=0}^{n-1} E f_{c}\left(Z_{i}\right)\left[h\left(Z_{i}\right)-h\left(Z_{n}\right)\right] .
\end{aligned}
$$

As in the derivation of $(6)$,

$$
\begin{aligned}
\sum_{i=0}^{n} E f_{c}^{2}\left(Z_{i}\right)+2 \sum_{i=0}^{n-1} E f_{c}\left(Z_{i}\right) h\left(Z_{i}\right) \\
=(n+1) E f_{c}^{2}\left(Z_{0}^{*}\right)+2 n E f_{c}\left(Z_{0}^{*}\right) h\left(Z_{0}^{*}\right) \\
\quad+r^{*}+O\left(\rho^{n}\right)
\end{aligned}
$$

where

$$
\begin{aligned}
r_{i}= & \sum_{i=0}^{\infty}\left\{E f_{c}^{2}\left(Z_{i}\right)-E f_{c}^{2}\left(Z_{0}^{*}\right)\right\} \\
& +2 \sum_{i=0}^{\infty}\left\{E f_{c}\left(Z_{i}\right) h\left(Z_{i}\right)-E f_{c}\left(Z_{0}^{*}\right) h\left(Z_{0}^{*}\right)\right\}
\end{aligned}
$$

Furthermore,

$$
\left|\sum_{i=0}^{\lfloor n / 2\rfloor} E f_{c}\left(Z_{i}\right) h\left(Z_{n}\right)\right|
$$




$$
\begin{aligned}
& \leq \sum_{i=0}^{\lfloor n / 2\rfloor}\left|E f_{c}\left(Z_{i}\right) P^{n-i} h\left(Z_{i}\right)\right| \\
& \leq \sum_{i=0}^{\lfloor n / 2\rfloor} E\left|f_{c}\left(Z_{i}\right)\right| \sup _{\substack{k \geq n / 2-1 \\
r \in S}}\left|E_{x} h\left(Z_{k}\right)\right| \\
& \leq n\|f\| O\left(\rho^{n / 2}\right) \\
& =O\left(\rho^{n / 4}\right)
\end{aligned}
$$

and

$$
\begin{aligned}
& \sum_{i=\lceil n / 2\rceil}^{n-1} E f_{c}\left(Z_{i}\right) h\left(Z_{n}\right) \\
& =\sum_{i=\lceil n / 2\rceil}^{n} E f_{c}\left(Z_{0}^{*}\right) h\left(Z_{n-i}^{*}\right)+O\left(\rho^{n / 2}\right) \\
& =E f_{c}\left(Z_{0}^{*}\right) k\left(Z_{0}^{*}\right)+O\left(\rho^{n / 2}\right)
\end{aligned}
$$

where

$$
k(x)=\sum_{j=0}^{\infty} E_{x} h\left(Z_{j}\right) .
$$

Combining (7) - (10) yields

$$
E\left(\sum_{j=0}^{n} f_{c}\left(Z_{j}\right)\right)^{2}=(n+1) \sigma^{2}+d+O\left(\rho^{n / 4}\right),
$$

where $\sigma^{2}=E f_{c}^{2}\left(Z_{0}^{*}\right)+2 E f_{c}\left(Z_{0}^{*}\right) h\left(Z_{0}^{*}\right)$, and $d=\kappa-$ $2 E f_{c}\left(Z_{0}^{*}\right) h\left(Z_{0}^{*}\right)-2 E f_{c}\left(Z_{0}^{*}\right) k\left(Z_{0}^{*}\right)$. We have therefore established the following result.

Theorem 1 If (5) holds, and $f$ is bounded, then A1 is satisfied.

The argument given above can be generalized substantially. As a consequence, we believe that the asymptotic result contained in proposition 1 holds in great generality in the discrete-event simulation setting. These results complement those of Whitt (1991); his analysis assumes that there exists a finite (deterministic) time $s$ at which $X$ is in stationarity, and that the simulationist has deleted all pre-s data from the estimator constructed.

\section{THE SENSITIVITY OF AN INITIAL TRANSIENT DETECTION TEST}

In this section, we study a test proposed by Schruben (1982) for detecting the presence of an initial transient in a steady-state simulation. It takes advantage of the fact that the (LT (2) can typically be strengthened to a functional CLT. Such a functional CLT demands that

$$
\frac{n^{1 / 2}}{\sigma}\left(\frac{1}{n} \int_{0}^{n t} X(s) d s-\alpha t\right) \Rightarrow B(t)
$$

as $n \rightarrow \infty$ ( $\Rightarrow$ here denotes weak convergence of stochastic processes), where $B=(B(t): t \geq 0)$ is a standard Brownian motion. If the initial transient is small, the idea is that the output series will then indeed be well approximated by the Brownian motion $B$ as implied by (11), so that the test statistic constructed from $X$ will have approximately the same distribution as that derived from $B$. On the other hand, if a significant initial transient is present, this will (hopefully) reflect itself in the test statistic taking on a value that is not representative of what is expected under $B$. Such unusual values of the test statistic then lead to a rejection of the hypothesis that the simulation has no initial transient. Schruben (1982) proposes a test statistic based on both the magnitude and location of the maximizer of a "standardized" version of the output series $\mathrm{X}$.

In this section, we will study the question of how large an initial transient must be present in order that test statistics derived from the output series will have corresponding distributions that are not well approximated by Brownian motion. This essentially comes down to the question of how large an initial transient must be present, in order that the functional CLT (11) break down.

It is difficult to analyze this question in full generality. Instead, our analysis focusses on a specific class of output processes that has qualitative structure representative of a large class of discrete-event simulations. Specifically, we shall be concerned with a discrete-time output series $\left(X_{n}: n \geq 0\right)$, in which the $X_{n}$ 's evolve according to an autoregressive relation of the form

$$
X_{n+1}=\rho X_{n}+V_{n+1}
$$

where $V=\left(V_{n}: n \geq 0\right)$ is the associated innovations sequence and $\rho \geq 0$. Because we shall later be interested in studying the effect of the autoregressive parameter $\rho$, we shall embed (12) in a family of autoregressions:

$$
X_{n+1}(m)=\rho_{m} X_{n}(m)+V_{n+1} .
$$

The simple dynamics of this system ensures that we can write $X_{n}(m)$ in terms of the innovation sequence, namely

$$
X_{n}(m)=\rho_{m}^{n} X_{0}(m)+\sum_{k=1}^{n} \rho_{m}^{n-k} V_{k}
$$


Consequently, assuming $\left|\rho_{m}\right|<1$,

$$
\begin{aligned}
\frac{1}{n} \sum_{j=0}^{\lfloor n t\rfloor} X_{j}(m)= & \frac{1}{n} \frac{\left(1-\rho_{m}^{\lfloor n t\rfloor+1}\right)}{1-\rho_{m}} X_{0}(m) \\
& +\frac{1}{n} \sum_{j=1}^{\lfloor n t\rfloor} V_{j} \sum_{k=0}^{\lfloor n t\rfloor-j} \rho_{m}^{k} .
\end{aligned}
$$

In order to proceed further, we need to impose some structure on the $I_{n}$ 's. Specifically, we will assume that the $V_{n}$ 's are i.i.d. r.v.'s for which $E V_{n}=\gamma$, $\operatorname{var} V_{n}=\eta$, and $E\left|V_{n}\right|^{2+\epsilon}<\infty$ for some $\epsilon>0$. Then, we can assume existence of a standard Brownian motion $B=(B(t): t \geq 0)$ such that

$$
\sum_{k=1}^{n} V_{k}=n \gamma+\eta B(n)+o\left(n^{1 / 2}\right) \quad \text { a.s. }
$$

as $n \rightarrow \infty$; (14) is known, in the literature, as a "strong approximation" for the $V_{n}$ 's. (See Csörgö and Révész (1981) and Philipp and Stout (1975) for additional details.)

Substituting (14) into (13) and simplifying, we get

$$
\begin{aligned}
\frac{n^{1 / 2}}{\sigma(m)} & \left(\frac{1}{n} \sum_{j=0}^{\lfloor n t\rfloor} X_{j}(m)-a(m) t\right) \\
= & \frac{B(n t)}{\sqrt{n}}+\frac{1}{\eta \sqrt{n}}\left(1-\rho_{m}^{n t}\right) X_{0}(m) \\
& -\frac{1}{\eta \sqrt{n}} \sum_{j=1}^{\lfloor n t\rfloor} V_{j} \rho_{m}^{n t-j+1}+o(1) \quad \text { a.s. }
\end{aligned}
$$

as $n \rightarrow \infty$, where $\alpha(m)=\gamma\left(1-\rho_{m}\right)^{-1}$ and $\sigma(m)=$ $\eta\left(1-\rho_{m}\right)^{-1}$. Noting that $B(n \cdot) / \sqrt{n}$ has the same distribution as does $B(\cdot)$, we see that the functional CLT (11) is valid so long as the other terms on the right-hand side of (15) converge to zero as $n \rightarrow \infty$. Given that the test of Schruben (1982) uses only values of $t \in[0,1]$, we note then that

$$
\frac{1}{\eta \sqrt{m}}\left(1-\rho_{m}^{m t}\right) Y_{0}(m) \rightarrow 0
$$

uniformly in $t \in[0,1]$, provided $X_{0}(m)=o(\sqrt{m})$. As for the other major term on the right-hand side of (15), we note that

$$
\begin{aligned}
& \sum_{j=1}^{\lfloor m t\rfloor} V_{j} \rho_{m}^{\lfloor m\lfloor\rfloor-j+1} \\
& \stackrel{\mathcal{D}}{=} \sum_{j=1}^{\lfloor m t\rfloor} V_{j} \rho_{m}^{j}=
\end{aligned}
$$

$$
\begin{aligned}
= & \left(1-\rho_{m}\right) \sum_{j=1}^{\lfloor m t\rfloor} V_{j} \sum_{k=j}^{\infty} \rho_{m}^{k} \\
= & \left(1-\rho_{m}\right) \sum_{k=1}^{\lfloor m t\rfloor} \rho_{m}^{k} \sum_{j=1}^{k} V_{j} \\
& +\rho_{m}^{\lfloor m t\rfloor} \sum_{j=1}^{\lfloor m t\rfloor} V_{j}^{r}\left(1-\rho_{m}\right) \\
= & \left(1-\rho_{m}\right) \sum_{k=1}^{\lfloor m t\rfloor} \rho_{m}^{k}(k \gamma+\eta B(k)+o(\sqrt{m})) \\
& +o(1) \quad \text { a.s. } \\
= & \left(1-\rho_{m}\right)^{-1} \gamma+o\left(\left(1-\rho_{m}\right)^{-1}\right) \quad \text { a.s., }
\end{aligned}
$$

where $\stackrel{\mathcal{D}}{=}$ denotes equality in distribution. Consequently, in order that

$$
\frac{1}{\sqrt{m}} \sum_{j=1}^{\lfloor m t\rfloor} V_{j} \rho_{m}^{m t-j+1}
$$

converge in probability to zero uniformly in $t \in[0,1]$, it must be that $m^{-1 / 2}\left(1-\rho_{m}\right)^{-1} \rightarrow 0$ as $m-\infty$. Hence, our analysis of the autoregressive model (12) leads to the following conclusion.

If the time horizon $m$ is large, we should not expect any test based on (11) to detect the presence of an initial transient unless either:

1. the initial condition $X_{0}$ of the autoregression (12) is of order $\sqrt{m}$ or larger, or

2. the auto-correlation parameter is such that $\rho$ is of the order $1-c / \sqrt{m}$ or larger.

Thus, this suggest.s that this class of tests can be effective for dealing with initial transients if either the autocorrelations in the simulation decay slowly, or if the initial condition for the simulation is significantly non- stationary; otherwise, the tests should not be cxpected to detect transient effects.

\section{ACKNOWLEDGEMENTS}

This work was supported by the Army Restarch Office under contract no. DAAL0:3-!)1-(i-(0)31!)

\section{REFERENCES}

Bratley, P., B. L. Fox, and L. E. Sihrage. 1987. A guide to simulation. Springer-Verlag, Now York. 
Csörgö, M. and P. Révész. 19\&1. Sitrong approximations in probability and statistics. Academic Press, New York.

Glynn, Peter W. 1984. Some asymptotic formulas for Markov chains with applications to simulation. Journal of Sitatistical Computation and Simulation 19:97-112.

Glynn, Peter W. 1987. Limit theorems for the method of replication. Stochastic Models 4:34:3-350.

Glynn, Peter Wr and D. L. Iglehart. 19!)(). Simulation output analysis using standardized timn series. Mathematics of Operations Research 15:1-16.

Lindvall, 'I. 1992. Lectures on the ('oupling Method. John Wiley, New York.

Meyn, S. P. and R. L. Tweedie. 1993. Markov chains and stochastic stability. Springer-Verlag, New York.

Philipp, W. and W. Stout. 1975. Almost sure invariance principles for partial sums of weakly dependent random variables. Mem. Amer. Math. Soc. No. 161 .

Schruben, L. W. 1982. Detecting initialization bias in simulation output. Operations Research 30:569590.

Whitt, W. 1991. The efficiency of one long run versus independent replications in steady-state simulation. Management Science 37:645-666.

\section{AUTHOR BIOGRAPHY}

PETER W. GLYNN received his Ph.D. from Stanford University, after which he joined the faculty of the Department of Industrial Engineering at the University of Wisconsin-Madison. In 1987, he returned to Stanford, where he currently holds the Thomas Ford Faculty Scholar Chair in the Department of Operations Research. He was a co-winner of the 1993 Outstanding Simulation Publication Award sponsored by the TIMS ( $r$ llege on Simulation. His research interests include discrete-event simulation, computational probability, queueing, and general theory for stochastir systrms. 